\title{
THE DYNAMICS OF THE FOREST GRAPH OPERATOR
}

\author{
Suresh Dara, S.M. Hegde \\ Department of Mathematical and Computational Sciences \\ National Institute of Technology Karnataka \\ Surathkal, Mangalore-575025, India \\ e-mail: suresh.dara@gmail.com \\ smhegde@nitk.ac.in \\ Venkateshwarlu Deva \\ S.B. RAO \\ C.R. Rao Advanced Institute of Mathematics, \\ Statistics and Computer Science \\ Hyderabad-500 046, India \\ e-mail: venky477@gmail.com \\ siddanib@yahoo.co.in
}

AND

Thomas ZaslaVsky

Department of Mathematical Sciences Binghamton University

Binghamton, NY, USA 13902-6000

e-mail: zaslav@math.binghamton.edu

\begin{abstract}
In 1966, Cummins introduced the "tree graph": the tree graph $\mathbf{T}(G)$ of a graph $G$ (possibly infinite) has all its spanning trees as vertices, and distinct such trees correspond to adjacent vertices if they differ in just one edge, i.e., two spanning trees $T_{1}$ and $T_{2}$ are adjacent if $T_{2}=T_{1}-e+f$ for some edges $e \in T_{1}$ and $f \notin T_{1}$. The tree graph of a connected graph need not be connected. To obviate this difficulty we define the "forest graph": let $G$ be a labeled graph of order $\alpha$, finite or infinite, and let $\mathfrak{N}(G)$ be the set of all labeled maximal forests of $G$. The forest graph of $G$, denoted by $\mathbf{F}(G)$, is the graph with vertex set $\mathfrak{N}(G)$ in which two maximal forests $F_{1}$, $F_{2}$ of $G$ form an edge if and only if they differ exactly by one edge, i.e., $F_{2}=F_{1}-e+f$ for some edges $e \in F_{1}$ and $f \notin F_{1}$.
\end{abstract}


Using the theory of cardinal numbers, Zorn's lemma, transfinite induction, the axiom of choice and the well-ordering principle, we determine the $\mathbf{F}$-convergence, $\mathbf{F}$-divergence, $\mathbf{F}$-depth and $\mathbf{F}$-stability of any graph $G$. In particular it is shown that a graph $G$ (finite or infinite) is $\mathbf{F}$-convergent if and only if $G$ has at most one cycle of length 3. The $\mathbf{F}$-stable graphs are precisely $K_{3}$ and $K_{1}$. The $\mathbf{F}$-depth of any graph $G$ different from $K_{3}$ and $K_{1}$ is finite. We also determine various parameters of $\mathbf{F}(G)$ for an infinite graph $G$, including the number, order, size, and degree of its components.

Keywords: forest graph operator, graph dynamics.

2010 Mathematics Subject Classification: Primary 05C76; Secondary $05 \mathrm{C} 05,05 \mathrm{C} 63$.

\section{REFERENCES}

[1] H.J. Broersma and X. Li, The connectivity of the leaf-exchange spanning tree graph of a graph, Ars Combin. 43 (1996) 225-231.

[2] R. Cummins, Hamilton circuits in tree graphs, IEEE Trans. Circuit Theory CT-13 (1966) 82-90. doi:10.1109/TCT.1966.1082546

[3] K.Ch. Das, A.S. Cevik and I.N. Cangul, The number of spanning trees of a graph, J. Inequal. Appl. 2013 (2013) article 395, 13 pages.

[4] K.Ch. Das, A sharp upper bound for the number of spanning trees of a graph, Graphs Combin. 23 (2007) 625-632. doi:10.1007/s00373-007-0758-4

[5] R. Diestel, Graph Theory, Third Edition, Graduate Texts in Mathematics, Volume 173 (Springer, Heidelberg, 2005).

[6] L. Feng, K. Xu, K.Ch. Das, A. Ilić and G. Yu, The number of spanning trees of a graph with given matching number, Int. J. Comput. Math. 93 (2016) 837-843. doi:10.1080/00207160.2015.1021341

[7] L. Feng, G. Yu, Z. Jiang and L. Ren, Sharp upper bounds for the number of spanning trees of a graph, Appl. Anal. Discrete Math. 2 (2008) 255-259. doi:10.2298/AADM0802255F

[8] G.R. Grimmett, An upper bound for the number of spanning trees of a graph, Discrete Math. 16 (1976) 323-324. doi:10.1016/S0012-365X(76)80005-2

[9] E. Kamke, Theory of Sets (Courier, 1950).

[10] J. Li, W.C. Shiu and A. Chang, The number of spanning trees of a graph, Appl. Math. Lett. 23 (2010) 286-290. doi:10.1016/j.aml.2009.10.006

[11] G. Liu, On connectivities of tree graphs, J. Graph Theory 12 (1988) 453-459. doi:10.1002/jgt.3190120318 
[12] E. Prisner, Graph Dynamics (CRC Press, 1995).

[13] J. Rodriguez and L. Petingi, A sharp upper bound for the number of spanning trees of a graph, in: Proceedings of the Twenty-Eighth Southeastern International Conference on Combinatorics, Graph Theory and Computing (Boca Raton, Fla., 1997), Congr. Numer. 126 (1997) 209-217.

[14] H. Shank, A note on Hamilton circuits in tree graphs, IEEE Trans. Circuit Theory CT-15 (1968) 86-86. doi:10.1109/TCT.1968.1082765

[15] D.V.V.P.R.V.B. Suresh, V. Deva and S.B. Rao, Dynamics of spanning tree graph operator, in: International Congress of Mathematicians ICM 2010, Short Communications Abstracts Book (Hindustan Book Agency, 2010) 472-473.

[16] Y. Teranishi, The number of spanning forests of a graph, Discrete Math. 290 (2005) 259-267.

doi:10.1016/j.disc.2004.10.014

[17] H. Whitney, 2-isomorphic graphs, Amer. J. Math. 55 (1933) 245-254. doi: $10.2307 / 2371127$

[18] F.J. Zhang and Z. Chen, Connectivity of (adjacency) tree graphs, J. Xinjiang University (Natural Science) 3 (1986) 1-5.

[19] X.-D. Zhang, A new bound for the complexity of a graph, Util. Math. 67 (2005) 201-203.

Received 31 July 2015

Revised 6 January 2016

Accepted 6 January 2016 